\title{
Trisomy 13 and 18: Selecting the Road Previously Not Taken
}

\author{
MARTIN J. MCCAFFREY*
}

\begin{abstract}
The care of patients with trisomy 13 and 18 is a source of significant controversy. While these conditions are life limiting, indisputable data refutes the notion that these conditions are lethal or incompatible with life. Despite such evidence, arguments of beneficence, quality of life and limited resources are invoked to make the case to limit care to trisomy children. Lessons learned in our ignominious history with Down syndrome should guide us as we explore care for patients with trisomy 13 and 18. As clinicians we should strive with equipoise to carefully examine available data, the current status of practices related to care from palliation to intensive interventions, rise above our personal prejudices and listen to the voices of families imploring us to consider their opinions regarding the value of the life of a child with trisomy 13 or 18 . We should recall and learn from our Down syndrome odyssey and select the road previously not taken as we chart a course to the best possible care for our trisomy 13 and 18 sisters and brothers. (c) 2016 Wiley Periodicals, Inc.
\end{abstract}

\section{How to cite this article: McCaffrey MJ. 2016. Trisomy 13 and 18: Selecting the road previously not taken. Am J Med Genet Part C Semin Med Genet 9999C:1-6.}

Vigorous discussions have been underway for several decades regarding the level of care to be provided individuals with trisomy 13 and 18 . This dialogue hearkens back to an era in which similar conversations were engaged regarding the care of infants with another chromosomal disorder, Down syndrome, later identified as trisomy 21. There are significant differences in the severity of some pathologies related to trisomy 13 and 18, but there have also been over the decades significant advances in medical and surgical care.

The reasons for the dramatic increase in the survival of infants with trisomy 21 seen over the last century are multifactorial. Survival clearly improved when generally accepted surgical management of a variety of surgical conditions associated with Down syndrome, commonly offered non-Down syndrome patients, was universally offered to Down syndrome patients. The literature documents significant delays in diagnosis of AV Canal, and failure to offer repair despite acceptable levels of pulmonary vascular resistance, persisting into the 1990s [Sondheimer et al., 1985; Amark and Sunnegårdh, 1999]. It was several high profile surgical cases, including the Baby Doe case, and controversial publications regarding withholding of care in NICUs, that led to political action spearheaded by parent and disability rights groups. These seismic societal shifts forced the medical community to extend commonly accepted medical interventions to Down syndrome patients [Mercurio, 2008].

The ongoing controversies regarding care to be offered infants with trisomy 13 and 18 in many ways mirrors our evolution as a medical community caring for individuals with trisomy 21 .
Until a confluence of events led to the regular application of surgical techniques to infants with trisomy 21 in the latter twentieth century, Down syndrome was characterized as life limiting, with survivors regarded as leading a life with little meaning and institutionalization encouraged. In 1949, Penrose posited a life expectancy of 8-12 years for individuals with Down syndrome [Penrose, 1949]. The terminology used to refer to trisomy 21 demonstrated general societal disregard for the value of these lives. Scientific literature through the 1970s demonstrates a slow progression in terminology from "defective mongoloids" to "mongoloids" to trisomy 21 and Down syndrome. Life expectancy is now as high as 55 years [Glasson et al., 2016]. As medical providers we have progressed from a point in the 1970 s at which $18 \%$ of providers advocated institutionalization

Dr. McCaffrey, M.D., CAPT USN (Ret), retired as a US Navy medical officer, neonatologist and advisor for neonatology to the Navy Surgeon General in 2006. He has been a member of the UNC Department of Pediatrics since 2006. He directs the Perinatal Quality Collaborative of North Carolina. He is a board member of the International Trisomy Alliance and has been a source of medical support for many families attempting to make difficult medical decisions in the care of their children with trisomy 13 and 18. He, and his wife Theresa, are the proud parents of Shea, their 15-year-old daughter with Down syndrome.

*Correspondence to: Prof. Martin J. McCaffrey, Division of Neonatal-Perinatal Medicine, Department of Pediatrics, UNC School of Medicine, Chapel Hill, NC 27544. E-mail: martin_mccaffrey@med.unc.edu

DOI 10.1002/ajmg.c.31512

Article first published online in Wiley Online Library (wileyonlinelibrary.com). 
for "mongoloids" to one at which we recognize our duty to understand and meet the unique medical needs of Down syndrome patients [Pueschel, 1985].

\section{The ongoing controversies} regarding care to be offered infants with trisomy 13 and 18 in many ways mirrors our evolution as a medical community caring for individuals with trisomy 21. Until a confluence of events led to the regular application of surgical techniques to infants with trisomy 21 in the latter twentieth century,

Down syndrome was characterized as life limiting, with survivors regarded as leading a life with little meaning and institutionalization encouraged.

The literature is replete with references to trisomy 13 and 18 as "lethal" conditions. So are trisomy 13 and 18 "lethal"? Wilkinson addressed this issue and identified the two most applicable interpretations of lethality. "Lethality" in this context refers either to "a condition that invariably leads to death either in utero or in the newborn period regardless of treatment" or "a condition that leads to death in utero or in the newborn period in most cases" [Wilkinson et al., 2014a]. The former definition is not relevant. The latter definition raises the question of what percentage of death is required to classify a condition as "lethal?" Meyer, in the largest population-based study of survival among children with trisomy 13 or trisomy 18 published to date, demonstrates 28 days and 1 year survivals of $25 \%$ and $12 \%$ for trisomy 13 , with $36 \%$ at 28 days and $13 \%$ at one year for trisomy 18. Meyer also notes significant variations in survival in the nine states included in the analysis. Survivals for trisomy 18 at 28 days and 1 year were $55 \%$ and $22 \%$ in Massachusetts; $49 \%$ and $24 \%$ in Georgia. Not only did Meyer demonstrate remarkable survival variation at one year, they also report that for infants alive at one year, survival to five years was $84 \%$ and $91 \%$ for trisomy 13 and 18 respectively [Meyer et al., 2016]. Survey data from Japan and the US, which includes large proportions of parents who elected to pursue medical interventions for their children with trisomy, report survivals of up to $50 \%$ at 1 year [Janvier et al., 2012a; Kosho et al., 2013].

So while the ability of significant numbers of trisomy infants to survive to one month, one year and beyond is clear, large segments of medical providers continue to describe trisomies 13 and 18 as "lethal" and "incompatible with life." Wilkinson surveyed obstetricians in Australia and New Zealand in 2013 and found that $85 \%$ believed trisomy 18 was lethal, 54\% felt trisomy 18 was incompatible with life, $78 \%$ did not believe a child with trisomy 18 could have a meaningful life but $77 \%$ felt that a child born with trisomy 18 and supported would have at least a 5-20\% 1 year survival [Wilkinson et al., 2014b]. In a survey of delivery room, community and childrens' hospital NICU nurses, pediatric residents and obstetric residents, Janvier reported that $71 \%$, $74 \%, 83 \%, 74 \%$, and $75 \%$, respectively would plan to terminate a pregnancy with a diagnosis of trisomy 18 [Janvier et al., 2012b]. Jacobs published on the attitudes of US neonatologists regarding trisomy 18 . The survey, while it suffers significantly from a $13 \%$ return rate, reports that $83 \%$ of neonatologists believe trisomy 18 is lethal, though only $60 \%$ feel that treatment of a child with trisomy 18 is futile, $45 \%$ believe the condition is incompatible with life, and $92 \%$ felt that survival of at least 5-20\% was likely at 1 year. Despite the significant possibility of survival at
1 year, only $16 \%$ felt such a life would be meaningful [Jacobs et al., 2016].

So while the ability of significant numbers of trisomy infants to survive to one month, one year and beyond is clear, large segments of medical providers continue to describe trisomies 13 and 18 as "lethal" and "incompatible with life."

What is one to make of these reports? Perhaps the best interpretation is that many providers accept that a life, as long as 1 year, is possible with trisomy 18 , but only a minority of providers see value in such a life. The unavoidable conclusion is that while most providers recognize that trisomy 13 and 18 are not lethal, they personally equate the life of a severely handicapped child with one of unjustifiable suffering. In labelling trisomy 13 and 18 lethal, it appears that some providers conflate their ability to assess quality of life with lethality. For many, death and severe handicap are indistinguishable.

McGraw in 2008 surveyed US neonatologists regarding whether they would initiate delivery room resuscitation in the care of a 36-week infant "with confirmed trisomy 18 and congenital heart disease." The heart disease in this vignette was not defined. Is this a VSD or hypoplastic left heart? It is curious that the authors give no indication that the type of cardiac defect has any bearing on the decisions to be made by respondents. Nevertheless, 44\% of respondents stated that they would initiate resuscitation, mainly at the request of the parents. The authors interpret this observation as the willingness of $44 \%$ of neonatologists to "abandon the best-interest standard" and adopt an "ethic of abdication." In their commentary the authors refer to 
trisomy 18 as lethal six times and state that "the vast majority of trisomy 18 infants die within the first month" (at odds with Rasmussen reporting 58\% mortality at one month for trisomy 18 in 2003 [Rasmussen et al., 2003]), that even with intensive support trisomy 18 infants "do not flourish" and "those who do live beyond the first year experience shortened lives marked by severe neurologic and physical impairment." The vignette construct and the author commentary are symptomatic of a prejudice that presumes an ability to determine that the quality of life to be lived by trisomy 18 infants is so dreadful that death is preferable and parents should not even be offered medical interventions for their child [McGraw and Perlman, 2008].

Graham [2016] offers a more comprehensive but similarly nihilistic view on the care of a child with trisomy 13 or 18 . While his title suggests he intends to make the case to limit cardiac surgery for trisomy patients with complex cardiac disease, Graham's essay is a broader appeal to deny consideration of all intensive care and cardiac surgery for trisomy infants. While there have been reports of success in cardiac surgery offered to trisomy patients, some patients certainly will suffer significant morbidity [Kosho et al., 2006; Kaneko et al., 2009]. Graham is on solid ground to advise caution in recommending surgery for complex cardiac defects, but he extends his recommendation to all cardiac lesions and intensive care. $\mathrm{He}$ cites three reasons: a failure to demonstrate effectiveness for such interventions, consumption of resources for "a patient without hope for a meaningful recovery," and quality of life and the best interest of the patient.

The efficacy of applying the same neonatal intensive care practices to trisomy infants that are offered to seriously ill infants with a normal karyotype has been shown to be effective. Kosho et al. [2006] reported a 1-year survival rate of $25 \%$ for trisomy 18 with intensive care. Several studies of trisomy support networks, while at risk for reporting bias, report enhanced survival with intensive care. Baty reported a 1-year survival rate of $42 \%$ for trisomy 18 infants (25\% were ventilated) and $38 \%$ for trisomy 13 patients (22\% were ventilated) [Baty et al., 1994]. Janvier et al. [2012a] reported a survival of 53\% at 1 year for trisomy infants receiving full intervention. It is worth noting that Graham, in citing the Kids' Inpatient database report from $\mathrm{Ma}$ which reports on the increased use of hospital services by children with trisomy 13 and 18 undergoing cardiac surgery, is also affirming that there are large numbers of trisomy patients regularly using hospital services [Ma et al., 2013]. Nelson in a larger evaluation of this data concluded, "Despite the conventional understanding of these syndromes (trisomy 13 and 18) as lethal, a substantial number of children are living longer than one year and undergoing medical and surgical procedures as part of their treatment" [Nelson et al., 2012].

\section{Kosho et al. reported a 1-year survival rate of $25 \%$ for trisomy 18 with intensive care. Several studies of trisomy support networks, while at risk for reporting bias, report enhanced survival with intensive care.}

To his credit, Graham openly states his ethical concerns with the consumption of resources by patients "without hope for a meaningful recovery." The question becomes one, however, of who will define what is a meaningful recovery? This is certainly a factor requiring consideration but we must be willing to accept our judgments in such matters are steeped in our own personal prejudices. If a decision to end a life is to be made based on quality of life and whether resources will be wasted on a life "without hope for a meaningful recovery", it would seem obligatory that we very seriously consider the input of parents of trisomy patients. Skotko has studied the views of parents of individuals with Down syndrome, demonstrating an overwhelming majority love and are proud of their child with Down syndrome. Only 4\% of respondents regretted having a child with Down syndrome. He also found that Down syndrome parents have a more positive outlook on life, stronger marital bond and their children are positively impacted by their sibling [Skotko et al., 2011].

While the possibility of severe neurocognitive impairment in their own child would be difficult for many medical providers to accept, the work of Janvier et al. [2012b] reveals that families living with trisomy 13 and 18 are similar to trisomy 21 families. This survey of parents of children with trisomy 13 or 18 , conducted in a social network, is edifying. Of respondent parents, $87 \%$ were told at some time after diagnosis that their child was incompatible with life, would live a life of suffering (57\%), would be a vegetable (50\%), would live a meaningless life (50\%), would ruin their marriage $(23 \%)$ or would ruin their family (23\%). Of families with a full trisomy child $40 \%$ lived more than a year and $21 \%$ lived at least 5 years. Of families whose children had died, $89 \%$ reported the overall experience of their child's life was positive. "Of the 159 parents whose child lived longer than 3 months, half stated that their child experienced more pain than other children and half recognized that caring for a special needs child was more difficult than they thought it would be. Yet, 98\% reported that this child enriched their life. Of the families in this cohort who had other children $(n=160), 82 \%$ felt that this child had a positive effect on siblings. When all parents were asked if they would continue the pregnancy if they discovered they were expecting another child with trisomy 13 or 18 , $8 \%$ responded negatively, 9\% were unsure, and $83 \%$ responded positively or said they would not do prenatal testing." Based on the data we have available from parents of children with trisomy 13 or 18 , there is little support for the view, held by many in the medical community, that no child with 
trisomy 13 or 18 should be offered the option of intensive care based on the presumption that they will have no quality of life, be an insufferable burden on their families and have no hope for meaningful outcome.

While the possibility of severe neurocognitive impairment in their own child would be difficult for many medical providers to accept, the work of Janvier reveals that families living with trisomy 13 and 18 are similar to trisomy 21 families. This survey of parents of children with trisomy 13 or 18, conducted in a social network, is edifying.

A number of single center studies attempt to examine the comparative effectiveness of "aggressive" or "intensive" care versus non-aggressive or palliative care in cases of trisomy 13 and 18 . The common pitfalls related to attempting to draw sound conclusions from such studies are illustrated in "Trisomy 18: A single-center evaluation of management trends and experience with aggressive obstetric or neonatal intervention." The authors report on 54 live births in this single center experience on the care of infants with trisomy 18 from 2004 to 2014 [Subramaniam et al., 2016]. This publication has been previously critiqued by Kosho and Carey [2016] but deserves further commentary. The authors report survival rates based on interventions they describe as "aggressive" and "nonaggressive." The groups included 36 and 13 infants respectively and the authors conclude, "Neither aggressive obstetric nor neonatal intervention increases survival of infants with T18 in our experience at a single center. These results should be used in counseling families with fetuses or newborns affected with T18."

The first flaw in this publication is that the authors do not have the statistical power to reasonably make this statement. The second difficulty is in delineating how many infants had "aggressive" care withdrawn after a postnatal trisomy diagnosis was made. It is common for "aggressive" care to be withdrawn after a postnatal trisomy diagnosis is made. Subramaniam reports that 40 infants were postnatally diagnosed. The report does not state how many of these infants initially had aggressive intervention, are categorized as such, and then had intervention withdrawn, leading to death, and skewing of comparisons. The third deficiency is in meeting the requirement that the "non-aggressive" and "aggressive" groups are comparable. There is no comparative reporting of key characteristics in the two treatment groups, but a comment is made regarding differences between the two groups related to a key element in survival, preterm birth. "It should be noted that there was no statistically significant difference in the mean gestational age at birth of infants in these two intervention groups (nonaggressive $38.0 \pm 1.6$ weeks vs. aggressive $36.7 \pm 3.3$ weeks; $P=0.06)$." A table summarizing individual patient data is included. Review of this table reveals a significant burden of prematurity in the deaths which occurred in the "aggressive" treatment group. There is also an overall trend to more severe prematurity in the "aggressive" group. Six infants $<33$ weeks were ventilated, included in the "aggressive" treatment group, and died. This disparity in prematurity in a report with such small patient numbers further handicaps any conclusions regarding comparisons of survival in the groups designated as "non-aggressive" and "aggressive."

A final consideration in counseling families regarding the prognosis and possible treatment plans for their child with trisomy 13 or 18 is our crude understanding of the relationship between trisomy genotypes and phenotypes. We classify patients with trisomy 13 and 18 as "full" or "mosaic" trisomy.
Based on lymphocyte karyotype studies, we regularly make predictions regarding outcomes which rely on this designation which disregards variability in phenotype and perpetuates notions of lethality. This phenotypic phenotypic variation in those infants we describe as "full trisomy" and "mosaics" is well described [Slavotinek et al., 2003; Hsu and Hou, 2007; Caba et al., 2013]. We are just beginning to appreciate the variable genetic expression that occurs in infants with Down syndrome who develop atrioventricular canal [Wang et al., 2016]. No doubt we will identify similar genotypic variabilities in infants diagnosed with trisomy 13 or 18 , and in the not too distant future our use of 5-30 cell lymphocyte analysis to karyotypically characterize patients will be considered primitive. More detailed genetic characterization of trisomy patients will ultimately allow us to develop a more sophisticated appreciation for the natural history of the multitude of expressions we once called full or mosaic trisomy. This will take us far in partnering with families as we develop management plans. For now, we should at least be willing to recognize the phenotypic reality that an infant with trisomy 13 or 18 and a diaphragmatic hernia or hypoplastic left heart faces much different challenges for survival than a child without those conditions.

The intent of this commentary is not to suggest that we can guarantee all children with trisomy 13 or 18 an extended life, nor that we should mandate resuscitation or all standard medical and surgical interventions for every trisomy infant. The intent is to plea for truth, transparency and recognition of our prejudices regarding the decisions we make and the care we offer patients with trisomy 13 and 18 . Parents have the right to be informed of the unique possibilities for their child, even if clinicians personally would not choose to actively support a child with trisomy 13 or 18. All families will not choose to medically intervene for their trisomy child, but some families, perhaps many, would welcome, with support, elements of this challenge. Subramaniam et al. state, "The natural history of trisomy 18 
is well studied, and our results are in keeping with the findings of others." A similar statement might have been made by a pediatrician regarding Down syndrome in 1960. As it was then for Down syndrome, given medical advancements, the tremendous variation in types of care offered trisomy 13 and 18 patients, and the commitment of many families to care for potentially technology dependent children with significant mental impairment, we must challenge Subramaniam's conclusion. Not only do we not fully understand the natural history of Trisomy 13 and 18 , our personal biases in predicting quality of life have limited our interest and willingness to explore this territory more fully.

The intent of this commentary is not to suggest that we can guarantee all children with trisomy 13 or 18 an extended life, nor that we should mandate resuscitation or all standard medical and surgical interventions for every trisomy infant. The intent is to plea for truth, transparency and recognition of our prejudices regarding trisomy 13 and 18 patients.

We have spent decades as medical providers labelling trisomy 13 and 18 infants as having a "fatal fetal malformation" which is "lethal" or "incompatible with life." While a short life is the fate for most children with trisomy 13 and 18 , it is a life. Each trisomy child is an individual with unique clinical characteristics. While there are commonalities regarding growth and neurologic outcomes for children with trisomy 13 or 18 , there is a diverse spectrum of associated medical conditions and potential life span. Labeling care as "aggressive" and "non-aggressive" with respect to all infants with trisomy illustrates our biases. Trisomy 13 and 18 are lethal in the main only to the point at which we limit intervention and decide that it is "aggressive". Such limitations can be applied to interventions that include standard obstetrical and newborn management, intensive care and provision of palliative care which shortens life rather than providing comfort, basic medical needs and the optimization of a shortened life, via medications or starvation. In this issue Janvier reports the disconcerting finding that palliative care for a prenatal diagnosis of trisomy 13 and 18 was different than that for a postnatal diagnosis. This survey of 332 parents in trisomy support networks revealed that care for a prenatal diagnosis of trisomy consisted mainly of warming with some use of sedative medications and no other interventions, whereas palliative care in postnatal diagnoses continued to include a variety of interventions. The authors found that the single most important factor independently related to mortality before discharge and at one year was the prenatal diagnosis. Janvier et al. are forced to conclude that the goal of palliative care provided after a prenatal trisomy diagnosis is as short a life as possible. These results should give us pause as providers to consider the roles our prejudices play in counseling families and treating their infants. All infants will not benefit from intervention, and many families will choose a palliative care course, but we should make such treatment decisions in transparent partnership with parents clearly stating our goals for care [Janvier et al., 2016].

Our partnerships with families should be predicated on the knowledge that we are speaking about a life changing diagnosis for a precious child and a family. This diagnosis does not diminish the value of the child's life, the child's value to the family, nor should we mistakenly believe we are called upon to do so. Our duty is to as comfortably as possible sustain life within the mutually agreed upon parameters that exist in the spectrum from palliation to intensive care. Our mission must be to openly engage families with the best available data, talk about their child with respect and dignity, discuss the unique medical conditions their child possesses, review the therapeutic options of palliative care and standard interventions, include specialists as required, openly recognize the potential harm interventions may carry, discuss long-term prognoses and make it clear that trisomy 13 and 18 are challenging, life limiting diagnoses generally characterized by severe cognitive and motor delays. We should be willing to offer support in parental decision making which includes the opportunity to speak with families who have cared for a trisomy 13 or 18 child. After this due diligence has taken place, we need to then determine what the wishes of the parents are and work with them collaboratively in attempting to achieve those goals.

As health care providers we ultimately blazed new trails over a century in the care we offer individuals with Down syndrome. On this voyage we gained a greater appreciation for the intrinsic and extrinsic value of the lives of these individuals. Decades later we are embarking on a similar odyssey in exploring the care we offer trisomy 13 and 18 patients. It is inaccurate to use "fatal fetal malformations", "lethal" and "incompatible with life" to describe to parents their infants with trisomy 13 and 18. As Lantos states, "These views are no longer tenable" [Boss et al., 2013]. History proves that pronouncements of lethality for these conditions will only insure one thing...continued lethality. We have much to learn about the natural history of trisomy 13 and 18, but these will remain uncharted waters unless we are willing to confront our biases and accept that many parents see an inherent dignity and value in the life of a child with trisomy 13 or 18 which some of us may not. In seeking the best approaches to the care of patients with trisomy 13 and 18 , let us select the road previously not taken in our ignominious Down syndrome journey. 


\section{REFERENCES}

Amark K, Sunnegårdh J. 1999. The effect of changing attitudes to Down's syndrome in the management of complete atrioventricular septal defects. Arch Dis Child 81:151154.

Baty BJ, Blackburn BL, Carey JC. 1994. Natural history of trisomy 18 and trisomy 13: I. Growth, physical assessment, medical histories, survival, and recurrence risk. Am J Med Genet 15 49:175-188.

Boss RD, Holmes KW, Althaus J, Rushton CH, McNee H, McNee T. 2013. Trisomy 18 and complex congenital heart disease: Seeking the threshold benefit. Pediatrics 132:161165.

Caba L, Rusu C, Butnariu L, Panzaru M, Braha E, Volosciuc M, Popescu R, Gramescu M, Bujoran C, Martiniuc V, Covic M, Gorduza EV. 2013. Phenotypic variability in Patau syndrome. Rev Med Chir Soc Med Nat Iasi 117:321-327.

Glasson EJ, Jacques A, Wong K, Bourke J, Leonard H. 2016. Improved survival in down syndrome over the last 60 years and the impact of perinatal factors in recent decades. J Pediatr 169:214-220.

Graham EM. 2016. Infants with trisomy 18 and complex congenital heart defects should not undergo open heart surgery. J Law Med Ethics 44:286-291.

Hsu HF, Hou JW. 2007. Variable expressivity in Patau syndrome is not all related to trisomy 13 mosaicism. Am J Med Genet A 1 143A:1739-1748.

Jacobs AP, Subramaniam A, Tang Y, Philips JB 3rd, Biggio JR, Edwards RK, Robin NH. 2016. Trisomy 18: A survey of opinions, attitudes, and practices of neonatologists. Am J Med Genet A 170:838-846.

Janvier A, Couture E, Deschenes M, Nadeau S, Barrington K, Lantos J. 2012a. Health care professionals' attitudes about pregnancy termination for different fetal anomalies. Paediatr Child Health 17:e86-e88.
Janvier A, Farlow B, Wilfond BS. 2012b. The experience of families with children with trisomy 13 and 18 in social networks. Pediatrics 130:293-298.

Janvier A, Farlow B, Barrington KJ. 2016. Hopes, interventions and survival of neonates with trisomy 13 and trisomy 18: What is palliative care for these conditions? Am J Med Genet $\mathrm{C}$ (in this issue).

Kaneko Y, Kobayashi J, Achiwa I, Yoda H, Tsuchiya K, Nakajima Y. 2009. Cardiac surgery in patients with trisomy 18 . Pediatr Cardiol 30:729-734.

Kosho T, Nakamura T, Kawame H, Baba A Tamura M, Fukushima Y. 2006. Neonatal management of trisomy 18: Clinical details of 24 patients receiving intensive treatment. Am J Med Genet A 140 9:937-944.

Kosho T, Kuniba H, Tanikawa Y, Hashimoto Y, Sakurai H. 2013. Natural history and parental experience of children with trisomy 18 based on a questionnaire given to a Japanese trisomy 18 parental support group. Am J Med Genet A 161A:1531-1542.

Ma MH, He W, Benavidez OJ. 2013. Congenital heart surgical outcomes in patients with trisomy 13 and 18. Circulation 128: A17120.

McGraw MP, Perlman JM. 2008. Attitudes of neonatologists toward delivery room management of confirmed trisomy 18: Potential factors influencing a changing dynamic. Pediatrics 121:1106-1110.

Mercurio MR. 2008. The aftermath of baby doe and the evolution of newborn intensive care. Georgia State University Law Rev 25:835863.

Meyer RE, Liu G, Gilboa SM, Ethen MK, Aylsworth AS, Powell CM, Flood TJ, Mai CT, Wang Y, Canfield MA; National Birth Defects Prevention Network. 2016. Survival of children with trisomy 13 and trisomy 18 A multi-state population-based study. Am J Med Genet A 170:825-837.

Nelson KE, Hexem KR, Feudtner C. 2012. Inpatient hospital care of children with trisomy 13 and trisomy 18 in the United States. Pediatrics 129:869-876.

Penrose LS. 1949. The incidence of mongolism in the general population. J Ment Sci 95:685688.

Pueschel SM. 1985. Changes of counseling practices at the birth of a child with Down syndrome. Appl Res Ment Retard 6:99-108.

Rasmussen SA, Wong LY, Yang Q, May KM, Friedman JM. 2003. Population-based analyses of mortality in trisomy 13 and trisomy 18. Pediatrics 111:777-784.

Skotko BG, Levine SP, Goldstein R. 2011. Having a son or daughter with Down syndrome: Perspectives from mothers and fathers. Am J Med Genet A 155A:2335-2347.

Slavotinek A, Poyser L, Wallace A, Martin F, Gaunt L, Kingston H. 2003. Two unique patients with trisomy 18 mosaicism and molecular marker studies. Am J Med Genet A 15 117A:282-288.

Sondheimer HM, Byrum CJ, Blackman MS. 1985. Unequal cardiac care for children with Down's syndrome. Am J Dis Child 139: 68-70.

Subramaniam A, Jacobs AP, Tang Y, Neely C, Philips JB 3rd, Biggio JR, Robin NH, Edwards RK. 2016. Trisomy 18: A singlecenter evaluation of management trends and experience with aggressive obstetric or neonatal intervention. Am J Med Genet 170A:838-846.

Wang L, Li Z, Song X, Liu L, Su G, Cui Y. 2016 Bioinformatic analysis of genes and MicroRNAs associated with atrioventricular septal defect in down syndrome patients. Int Heart J 57:490-495.

Wilkinson DJ, de Crespigny L, Lees C, Savulescu J, Thiele P, Tran T, Watkins A. 2014a. Perinatal management of trisomy 18: A survey of obstetricians in Australia, New Zealand and the UK. Prenat Diagn 34:42-49.

Wilkinson D, de Crespigny L, Xafis V. 2014b. Ethical language and decision-making for prenatally diagnosed lethal malformations. Semin FNeonatal Med 19:306-311. 